\title{
Antibiotics' administration and their association with adverse drug reaction in public pediatric clinics of Cyprus
}

\author{
Evripidou Iliada1; Evripidou Andri'; Koliou Maria1; Latsou Dimitra1; Geitona Mary ${ }^{1}$ \\ ${ }^{1}$ University of Peloponnese, Corinth, Greece
}

Background: Therapy with antibiotics is a cornerstone in clinical practice, however the overuse of antibiotics is a serious public health problem (1). Antibiotics are frequently administered to pediatric patients and are responsible for most of adverse drug reactions (ADRs). Additionally, antibiotics are reported as the most troublesome of drugs contributing to approximately $15 \%$ $16 \%$ of cases of ADRs (2).

Aim: The aim of this study was to detect the ADRs incidence related to the administration of antibiotics in public pediatric clinics of Cyprus.

Methods: A cross-sectional study was carried out over a two-month period (April-May 2015) in all public pediatric clinics. Data collection was realized with a selfadministered questionnaire, which was distributed to 57 in total physicians providing pediatric care in public hospitals. Descriptive statistics and non-parametric tests were performed to determine the significant differences between antibiotic administration and the ADRs incidence.

Figure 1: Administration of antibiotics in common childhood diseases
Results: In total 42 out of 57 pediatricians filled out the questionnaire $(\mathrm{RR}=74 \%) . \quad 66.7 \%$ mentioned that implement the "watchful waiting" approach in acute otitis media and 59.5\% administered often/very often antibiotics therapy in pharyngitis. Nevertheless $85.7 \%$ rarely/never administer antibiotics for the prevention of secondary respiratory tract infections (Fig 1). 23.1\% declared often/very often ADRs incidence after antibiotic administration. Statistically significant differences were observed between the frequency of antibiotics administration in pharyngitis and in the prevention of secondary respiratory tract infections related to the ADRs incidence (Table 1).

Table 1: Frequency of antibiotics administration (often / very often) related to the ADRs incidence

\begin{tabular}{|l|c|c|c|}
\hline \multirow{2}{*}{} & \multicolumn{2}{|c|}{ ADRs incidence } & \multirow{2}{*}{ P value } \\
\cline { 2 - 3 } & $\begin{array}{c}\text { Rarely } \\
\text { /never }\end{array}$ & $\begin{array}{c}\text { Often / } \\
\text { very often }\end{array}$ & \\
\hline Pharyngitis & $68.2 \%$ & $31.8 \%$ & 0.080 \\
\hline $\begin{array}{l}\text { Prevention of } \\
\text { secondary } \\
\text { respiratory tract } \\
\text { infection }\end{array}$ & $60.0 \%$ & $40.0 \%$ & 0.026 \\
\hline
\end{tabular}

Often / very often Rarely /never No answer

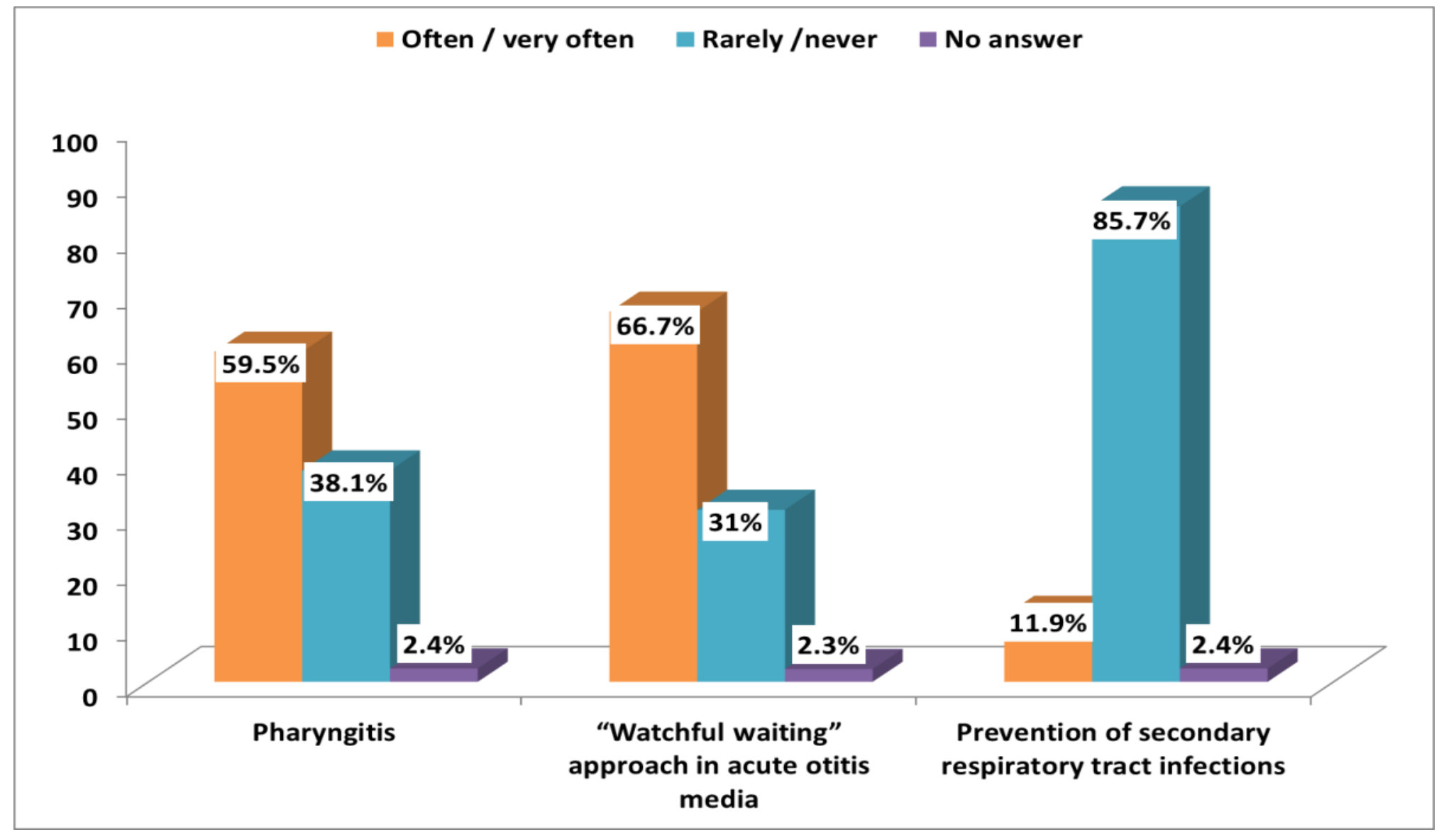

Conclusions: Therapeutic guidelines and protocols focused on pediatric care are necessary to be introduced in the everyday clinical practice in all public hospitals in Cyprus. Physicians' continuing education on the appropriate antibiotics' administration as well as patients' awareness on their negative impact on health are also required. 